\title{
A Comparison of Techniques for PET Data-Driven Tracking of Head Motion
}

Ashley Gillman ( $\square$ Ashley.Gillman@csiro.au )

Commonwealth Scientific and Industrial Research Organisation https://orcid.org/0000-0001-91301092

\section{Stephen Rose}

Commonwealth Scientific and Industrial Research Organisation

Jye Smith

Queensland Health

Jason A Dowling

Commonwealth Scientific and Industrial Research Organisation

Nicholas Dowson

Optellum

\section{Research Article}

Keywords: motion correction, PET, data-driven tracking, head motion

Posted Date: February 17th, 2021

DOI: https://doi.org/10.21203/rs.3.rs-202051/v1

License: (c) (i) This work is licensed under a Creative Commons Attribution 4.0 International License.

Read Full License 


\section{A comparison of techniques for PET data-driven tracking of head motion}

Ashley G. Gillman, ${ }^{\circledast 2}$, Stephen Rose ${ }^{1,2}$, Jye Smith $^{5,6}$, Jason A. Dowling ${ }^{1}$, and Nicholas Dowson $\dagger^{\dagger 7}$

${ }^{1}$ Australian e-Health Research Centre, Commonwealth Scientific and Industrial Research Organisation, Brisbane, Australia

${ }^{2}$ Faculty of Medicine, University of Queensland, Brisbane, Australia

${ }^{5}$ Department of Nuclear Medicine and Specialised PET Services, Royal Brisbane and

Women's Hospital, Brisbane, Australia

${ }^{6}$ Herston Imaging Research Facility, Royal Brisbane and Women's Hospital, Brisbane, Australia

${ }^{7}$ Optellum, Oxford, United Kingdom

February 3, 2021

Running title: PET data-driven tracking of head motion Mr Gillman is financially supported by an Australian Government Department of Education and Training Research Training Program stipend and a Commonwealth Scientific and Industrial Research Organisation (CSIRO) Top Up Scholarship.

Word count: 3382 words

\footnotetext{
${ }^{*}$ Corresponding author. email: ashley.gillman@csiro.au phone: +61404085204

Level 5 UQ Health Sciences Building, RBWH Campus

Brisbane 4029

Australia

${ }^{\dagger}$ Senior author
} 


\begin{abstract}
Background / Aims: Patient motion during positron emission tomography (PET) imaging can corrupt the image by causing blurring and quantitation error due to misalignment with the attenuation correction image. Data-driven techniques for tracking motion in PET imaging allow for retrospective motion correction, where motion may not have been prospectively anticipated.

Methods: A two minute PET acquisition of a Hoffman phantom was acquired on a Biograph $\mathrm{mCT}$ Flow, during which the phantom was rocked, simulating periodic motion with varying frequency. Motion was tracked using the sensitivity method, the axial centre-of-mass (COM) method, a novel 3D-COM method, and the principal component analysis (PCA) method. A separate two minute acquisition was acquired with no motion as a gold standard. The tracking signal was discretised into 10 gates using k-means clustering. Motion was modelled and corrected using the reconstruct-transform-add (RTA) technique, leveraging Multimodal Image Registration using Block-matching and Robust Regression (Mirorr) for rigid registration of nonattenuation-corrected 4D PET and Software for Tomographic Image Reconstruction (STIR) for PET reconstructions. Evaluation was performed by segmenting white matter (WM) and grey matter (GM) in the attenuation correction computed tomography (CT). The mean uptake in the region of GM was compared with that in the WM region. Additionally, the difference between the intensity distributions of WM and GM regions was measured with the t-statistic from a Welch's t-test.

Results: Difference in the mean distribution of WM to GM ranked the techniques in order of efficacy: no correction, sensitivity, axial-COM, 3D-COM, PCA, no motion. PCA correction had a great WM/GM separation measured by the t-value than the no motion scan. This was attributed to interpolation blurring during motion correction reducing class variance.

Conclusion: Of the techniques examined, PCA was found to be most effective for tracking rigid motion. The sensitivity and axial-COM techniques are mostly sensitive to axial motion, and so were ineffective in this phantom experiment. 3D-COM demonstrates improved transaxial motion sensitivity, but not to the level of effectiveness of PCA.
\end{abstract}

(324 words)

Keywords: motion correction, PET, data-driven tracking, head motion 


\section{Introduction}

Patient motion during neurological positron emission tomography (PET) corrupts images, causing blurring and quantitation error due to misalignment with an attenuation correction image. These effects result in poorer image quality, which may affect patient management in clinical practice, or reduce effect size in research. For these reasons, motion correction has been employed to reduce the effect of patient motion.

In neurological imaging, it can be difficult to predict before a scan whether motion will occur. Many subjects can remain still and motion correction is only required in a subset of scans. Motion correction techniques can require complex hardware to be set up before a scan, which can be impractical in clinical practice due to the time taken to setup the apparatus, especially for centres where the PET scanner is in high demand. To address this, some authors have proposed a class of techniques which can track motion from raw PET data, without requiring additional hardware. These techniques are called data-driven motion tracking techniques.

Various techniques for data-driven PET motion tracking have been proposed, and are described in Section 2. In this work, a Hoffman phantom acquisition, described in Section 3.1, contrasts the subset of these techniques that are able to be employed for tracking head motion. The applicable techniques used in this investigation are described in more detail in Section 3.2. Some of the other techniques that are not applicable in this investigation are addressed briefly in the discussion. These signals are used to perform motion correction of a phantom acquisition, as described in Section 3.3 and the results are objectively ranked by comparing white matter (WM) and grey matter (GM) voxel distributions, as described in Section 3.4. Similar previous works either are not comprehensive in evaluating the applicable techniques for head motion [1, 2], or are focused on thoracic or abdominal motion [3] - the authors believe this work is the first to focus on this niche in a phantom experiment.

\section{Background}

Various methods for data-driven motion correction have been developed, and those applicable to head motion are investigated in this work. Data-driven techniques analyse the distribution of raw PET data, in order to determine when motion occurs. They produce a motion surrogate, a signal related to motion that can be used for correction. 
The first of such techniques proposed was the sensitivity technique [4, 5, 6], originally proposed by Visvikis et al. [4]. This method exploits the fact that the sensitivity profile of the scanner is heterogeneous. In a typical cylindrical PET gantry, the sensitivity varies axially, approximately linearly with axial distance from the isocentre of the gantry rings, with the most sensitive region being in the centre of the PET gantry. There are also smaller sensitivity variations transaxially, but the scanner is more sensitive to axial changes that transaxial. These are depicted in Fig. 1. Patient motion causes a shift of the emission distribution with respect to the static sensitivity distribution, resulting in a change in the instantaneous count rate. Hence, the instantaneous count rate can be used as a motion surrogate, and has been applied to respiratory motion, where it tracks the diaphragm. The diaphragm moves in the craniocaudal direction, parallel to the axial direction of the PET scanner where changes in sensitivity are most pronounced and hence where motion is most easily detected. The method can also be applied to head motion. Extensions to this technique that mask out regions without motion to improve signal-to-noise ratio (SNR) have been proposed [7]. Spectral techniques are able to identify periodic motion, such as respiratory or cardiac motion, and count rates are only calculated in the regions that have not been masked out. These techniques are not investigated in this work, as they are not applicable to head motion.
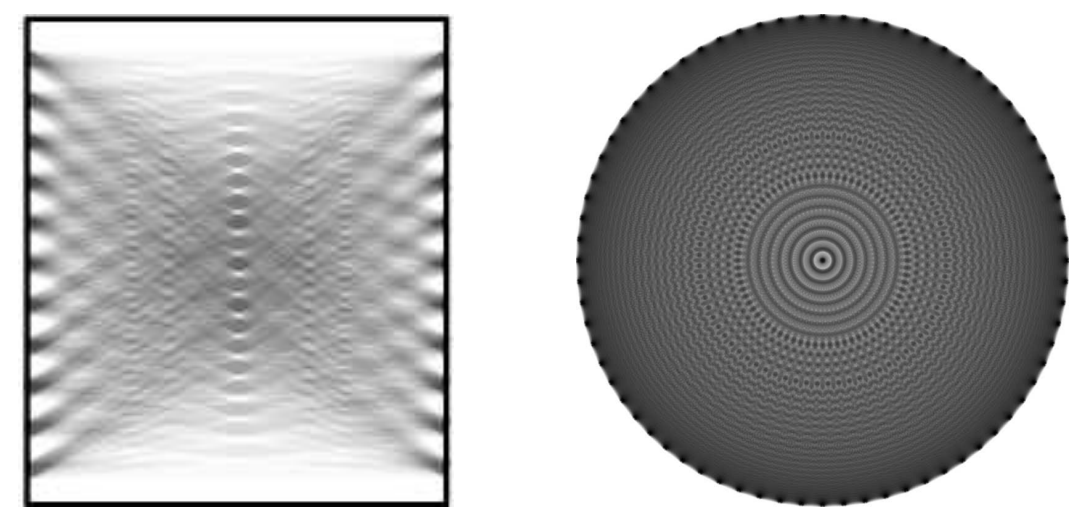

Figure 1: Left) Top/axial view, with the gantry axis running vertically, and Right) front/transaxial view of a cylindrical PET gantry. Lines of responses are shaded in with opacity so that darkness in the image approximates the spatial sensitivity.

The axial-centre-of-mass $(\mathrm{COM})$ technique [8] is calculated similarly to the previous technique, but instead of measuring count rates, the central tendency of the distribution is measured. Specifically, it measures the axial centre-of-mass (COM) of raw data sinograms. In tomography, the axial coordinate in sinogram and image space is identical, therefore the COM in raw data space corre- 
sponds to the COM axially of the emission image. So this motion surrogate measures the mean $z$ position of the distribution, yielding an interpretable value with units of distance. As with the sensitivity techniques, spectral analysis masking extensions are available [9], but are not investigated in this work as head motion is the focus.

Ren et al. [10] introduced a three dimensional extension to the COM technique called centroidof-distribution (COD) [11]. Each detected event has an associated emission location probability distribution that corresponds to the probability an emission at a given location caused the event. When time of flight (ToF) information is included, the distribution can be reduced to a relatively tight distribution, with the mode of the distribution representing the most likely emission location. In the COD and related [1, 12] surrogate extraction techniques, the modal emission location for each event is recorded, and the centroid of these in a given time period represents the centre of the distribution being tracked. This exact technique was not examined here, because it relies upon ToF information to work, which was not the focus of this investigation. Instead, an alternative 3D extension of the axial COM technique, proposed in Section 3.2, was examined.

The final method investigated is the principal component analysis (PCA) technique [13, 2], which uses principal component analysis to define a linear model of the modes of variance in the raw data histogram intensities. A truncated PCA transformation [14] yields a dimensionality reduced signal, each component of which measures an independent change in the estimated true count distribution over time. The primary modes of variation encode gross structured changes. Assuming such structured (i.e., non-noise) temporal changes do not occur to factors such as tracer kinetics, then the output of a PCA transform will correspond to patient pose from which relative motion can be inferred.

Commonly, this motion surrogate is used to divide the acquisition into a number of approximately motion-free segments, either as a series of frames, or periodic gates. Motion correction can then be applied, for example, using the reconstruct-transform-add (RTA) motion correction scheme [15, 16]. In this scheme the measured pose transform for each gate is applied to the attenuation coefficient image ( $\mu$-map) in order to get an aligned $\mu$-map. Each gate is then independently reconstructed, and the inverse pose transform applied to correct the reconstruction back to the reference pose. Finally, the gates are summed to estimate the total activity with a high SNR. 


\section{Methodology and materials}

\subsection{Acquisition}

A Hoffman phantom was filled with $65 \mathrm{MBq}$ of ${ }^{18}$ F-Fluorodeoxyglucose (FDG). For the test data, a two minute PET acquisition was acquired on a Biograph mCT Flow. During the first $30 \mathrm{~s}$ of the acquisition, the phantom was kept still. During the remainder of the scan, the phantom was manually rocked in the scanners left-right axis, simulating periodic motion with a period of approximately $2 \mathrm{~s}$. In addition, a two minute PET acquisition was acquired in which no motion occurred, as a gold-standard. At the beginning of each scan, a computed tomography (CT) was acquired for attenuation correction. The corresponding $\mu$-map was created with Siemens' software, using their proprietary multi-linear scaling technique of the Houndsfield unit (HU) intensities.

\subsection{Surrogate extraction}

Four data-driven PET motion tracking techniques were investigated for motion surrogate extraction, the sensitivity method [5, 6], the axial-COM method [8], a modified 3D-COM method, and the PCA method 13,2$]$.

The equation for the sensitivity surrogate, $\tau_{\text {sens }}$, is given in equation Eq. (1). $T$ is the true event distribution in sinogram space, and is indexed by $e, a, z, s$, and $t$, the radial position, angle, axial position, segment and time, respectively. $T=P-D$, where $P$ and $D$ are the prompt and delayed event distributions. At each time point, $t$, the resulting true counts are summed over the domains of $e, a, z$ and $s$, respectively $E, A, Z$ and $S$, yielding the total estimated true counts at $t$. The signal is decay corrected using the radioisotope half life, $\lambda$.

$$
\tau_{\mathrm{sens}}(t)=e^{\lambda t} \sum_{e, a, z, s \in E, A, Z, S} T_{e, a, z, s, t}
$$

The second method investigated is the axial-COM surrogate, $\tau_{\mathrm{COMz}}$. The equation used to determine the motion surrogate signal is a weighted mean of counts, weighted by the axial position, and is given in Eq. 22.

$$
\tau_{\mathrm{COMz}}(t)=\frac{\sum_{e, a, z, s \in E, A, Z, S} z T_{e, a, z, s, t}}{\sum_{e, a, z, s \in E, A, Z, S} T_{e, a, z, s, t}}
$$


In the third method investigated, the axial-COM surrogate is extended to three dimensions, as an alternative to the COD technique. To achieve this, an approximately parallel subset of the transaxial lines of response (LORs) is considered. The COM of the vertical lines gives the mean $x$ location of the distribution, and the COM of the horizontal lines give the mean of the mean $y$ location of the distribution, as depicted in Fig. 2. This technique avoids ascribing a position to each count in the direction parallel to the LOR, which has the most uncertainty without ToF information.

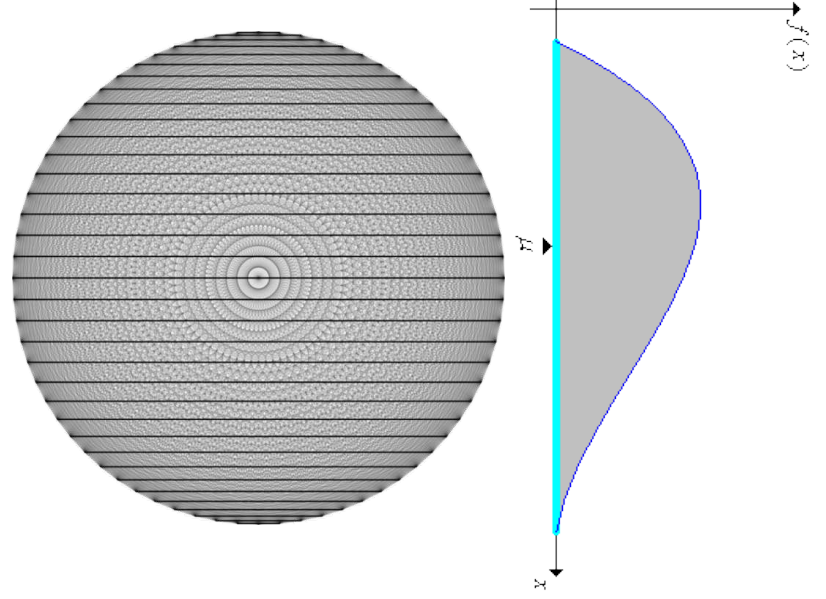

Figure 2: An axial view of a gantry, with LORs shown in grey and horizontal LORs highlighted in black. The distribution of counts of the horizontal LORs gives an indication of the vertical distribution of the emission distribution.

The formulae are given in Eq. (3). $A_{x}$ is the set of approximately horizontal angle samples, defined as $A_{x}=\{a \in A \mid 0 \leq a<m\}$, where $m$ is the range of angles considered parallel, here defined as $7.143^{\circ}$, corresponding to 10 angular sample points in the sinogram. $A_{y}$ is the set of approximately vertical angle samples, $A_{y}=\left\{a \in A \mid 90^{\circ} \leq a<90^{\circ}+m\right\}$.

$$
\begin{gathered}
\tau_{\mathrm{COMx}}(t)=\frac{\sum_{e, a, z, s \in E, A_{y}, Z, S} e T_{e, a, z, s, t}}{\sum_{e, a, z, s \in E, A_{y}, Z, S} T_{e, a, z, s, t}} \\
\tau_{\mathrm{COMy}}(t)=\frac{\sum_{e, a, z, s \in E, A_{x}, Z, S} e T_{e, a, z, s, t}}{\sum_{e, a, z, s \in E, A_{x}, Z, S} T_{e, a, z, s, t}} \\
\tau_{\mathrm{COMz}}(t)=\frac{\sum_{e, a, z, s \in E, A, Z, S} z T_{e, a, z, s, t}}{\sum_{e, a, z, s \in E, A, Z, S} T_{e, a, z, s, t}}
\end{gathered}
$$

The PCA surrogate, $\tau_{\mathrm{PCA}}$, was calculated similarly to that proposed by Thielemans et al. [13]. 
An activity mask is estimated by median filtering and thresholding at $10 \%$ of the 95 th percentile activity the static sinogram. The mask was applied to the estimate true series, $T$, which was subsequently normalised to have equal counts at each time point (obviating the need for decay correction), then variance stabilised with a Freeman-Tukey transform [17], yielding $T_{\text {norm. }}$ A PCA was conducted on $T_{\text {norm }}$ to determine the PCA weights, $W$, and the surrogate is given by the transform to $k$ components, given in Eq. (4). In this work, 2 components were found to be sufficient to capture motion, after observing the signals, yielding a 2 dimensional PCA surrogate.

$$
\tau_{\mathrm{PCA} k}(t)=T_{\text {norm } t} \cdot W_{k}
$$

\subsection{Motion correction}

For each surrogate extraction method, the motion surrogate is generated using the PET list mode data, and k-means clustering with 10 groups is used to discretise the surrogate into a gating signal. Although k-means is an unconventional motion surrogate discretisation technique, it allows for clustering of multidimensional signals (PCA and 3D-COM) in an identical manner to single dimensional signals (sensitivity, axial-COM). A motion model is formed by binning the list mode data into 10 separate sinograms, each containing data from one of the 10 gates from the gating signal, generating a non-attenuation-corrected (NAC) PET reconstruction for each, and finally registering each to a chosen reference gate using an iterative registration scheme. Reconstructions were performed using Software for Tomographic Image Reconstruction (STIR).

The iterative registration scheme, depicted in Fig. 3, ensured high quality registrations, even when some gates may have low counts contributing to the reconstructions and hence have a low SNR. A sum over all frames reduces the SNR with some additional blur due to motion, and is registered to the $\mu$-map to ensure the accuracy of subsequent attenuation correction, and to prevent the registrations from drifting through iterations. Each individual frame is then registered to the aligned, summed image. Registering frames to this image is more accurate than registering directly to the 1 -map, as the mono-modality registration is simpler to optimise. These steps are iterated three times, so that the sum image is less blurred due to motion at each step. The Multimodal Image Registration using Block-matching and Robust Regression (Mirorr) [18] software was used for rigid registrations, with the normalised correlation metric, omitting the highest resolution step 
of the resolution pyramid.

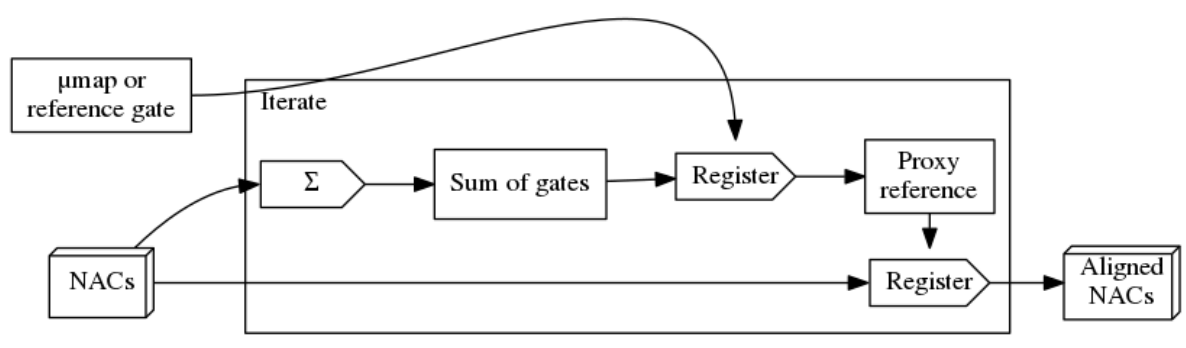

Figure 3: Iterative PET gate registration scheme. Square and cube shaped boxes indicate images and image series respectively. Pentagonal boxes indicate processes. In registration processes, the top input is the fixed/reference image, and the left input is the moving image. The portion of the workflow within the iterate box is repeated.

Motion correction was subsequently performed using the RTA scheme. In this scheme, PET data from each gate or frame is reconstructed separately without attenuation correction. These reconstructions are registered with a reference to determine pose parameters at each gate. Next, the measured pose transform for each gate is applied to the $\mu$-map in order to get an aligned $\mu$-map. Each gate is then independently reconstructed, and the inverse pose transform applied to correct the reconstruction back to the reference pose. Finally, the gates are summed to estimate the total activity with a high SNR.

\subsection{Analysis}

A novel technique is proposed to objectively and quantitatively analyse motion correction in the image. The CT was segmented by intensity into WM and GM classes. The segmentation is used to mask GM and WM PET voxels and generate a distribution of PET intensity in each tissue. Blurring due to uncorrected motion causes higher PET activity in the GM to spill into the WM, causing the two distributions to converge. Therefore, the difference between the distributions increases with motion blur. The difference between the two distributions is measured with the difference in means, and with the t-statistic from a Welch's t-test. Each measure provides an objective and quantitative surrogate measure of motion corruption. 


\section{Results}

The extracted motion surrogates are depicted in Fig. 4. The figure also shows the gating signal, with each gate illustrated as the hue of each point. Since no motion occurred during the first 30 $\mathrm{s}$ of acquisition, the variance in this portion of the signal is low. Comparing the signal variance in the remaining portion with this portion allows an appreciation of the approximate SNR of each technique.

Although some signal can be seen in the surrogate for the sensitivity technique, without extensive filtering, requiring a priori knowledge about the motion, the SNR is too low for good estimation of pose. The axial-COM technique provides an improved signal. Of the three 3D-COM components, the $\mathrm{x}$ component measured most of the motion signal, which is consistent with the motion applied to the phantom. This indicates that the 3D-COM should provide superior gating. Finally, the first component of the PCA surrogate exhibits very low variation during the no-motion portion of the scan, and clear periodic motion during the remainder. The second component of the PCA captures a signal with twice the period of the first, consistent with capturing the vertical component of the rocking.

Fig. 5 shows axial slices through each of the reconstructed images. Images have been ordered by increasing subjective quality of motion correction, which was determined to be no correction, sensitivity, axial-COM, 3D-COM, PCA, no motion. Fig. 6 shows the objective analysis results outlined in Section 3.4 in the same order. This figure depicts the results of the t-test and the difference between distribution means.

\section{Discussion}

\subsection{Subjectively, PCA extracts lowest noise motion signals}

Subjective estimation of SNR of the raw motion surrogates (Fig. 4 agreed with the subjective analysis of motion correction quality (Fig. 5). The sensitivity technique provided some improvement compared to doing no correction, although the improvement is only slight. Although the motion surrogate for axial-COM appeared better that the sensitivity surrogate, the improvements seen in the reconstructed image are limited (Fig. 4). The 3D-COM technique exhibits less blurring than 



Figure 4: Plot of extracted surrogates. Surrogates have been gated, indicated by colour (online version only), using k-means gating. 

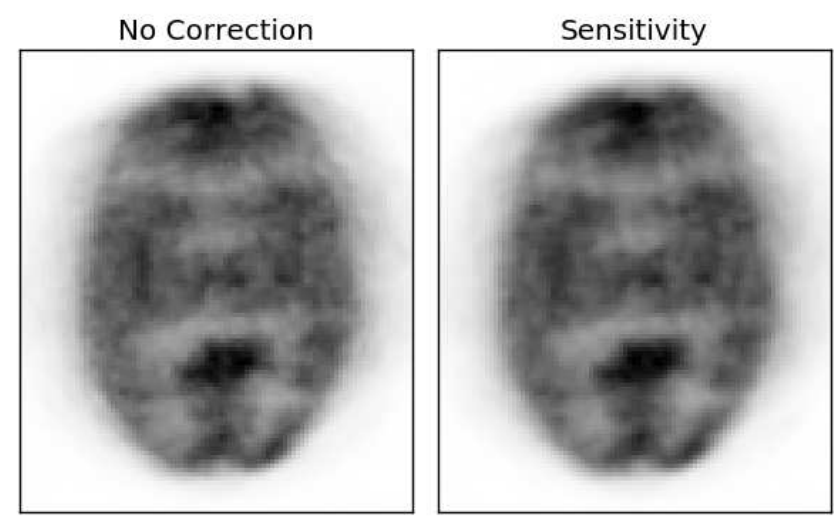

PCA
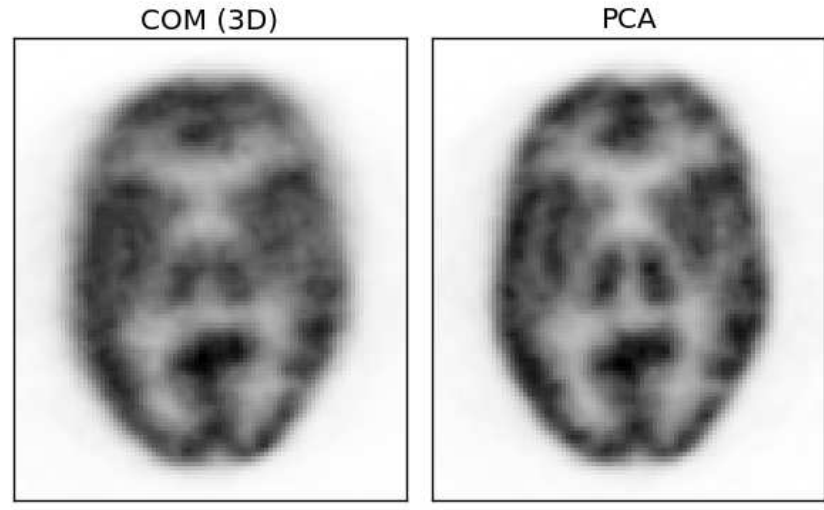

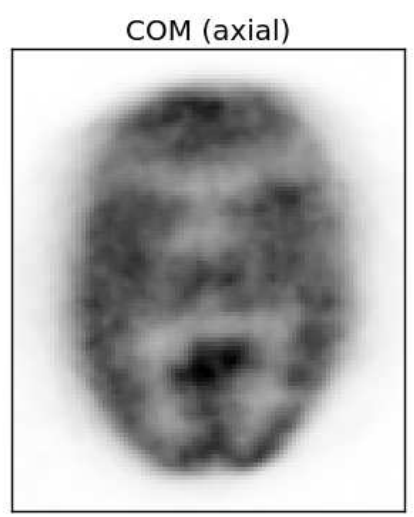

No Motion (gold)

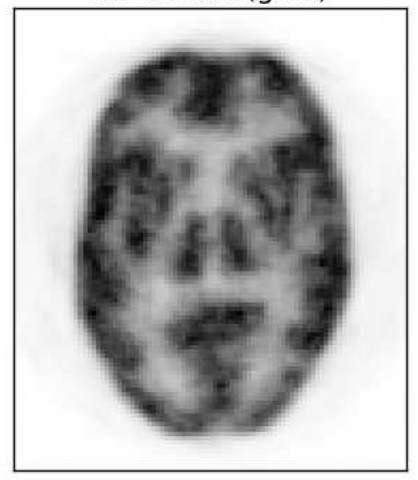

Figure 5: Axial slice of reconstructions with each of the investigated tracking techniques, as well as a reconstruction with no correction and a reconstruction of the reference acquisition of the phantom with no induced motion. 


\section{Grey matter - white matter voxel intensity distributions}

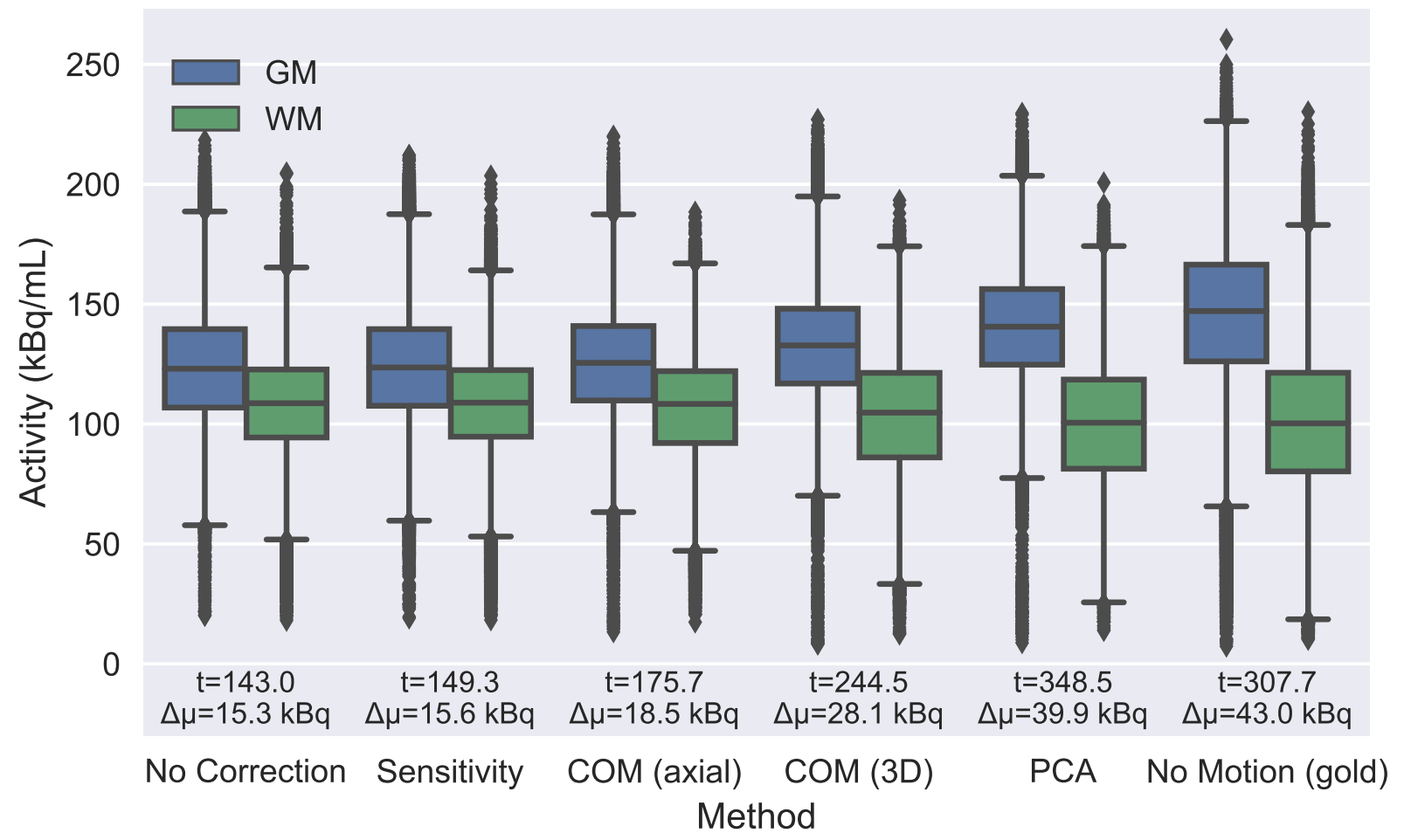

Figure 6: Distributions of activity in voxels labelled GM (left and blue online) and WM (right and green online) in the CT segmentation, for each correction method investigated. Shown below each pair of distributions is the t-statistic between them $(t)$ and the difference in the distribution means $(\Delta \mu)$. A larger metric implies a greater separation between classes, indicating less blurring. 
axial-COM. The 3D-COM surrogates (Fig. 4 show that most motion occurred in the $\mathrm{x}$ direction, and only a small change was measured in the $\mathrm{z}$ direction. This explains the lower performance of the sensitivity and axial-COM techniques, which are mostly sensitive to axial motion. Finally, the reconstructions using the PCA technique approach appearing similar to a smoothed version of the no motion reconstruction. As expected, reconstructing and applying no motion correction resulted in the lowest subjective image quality, and acquiring data with no motion present resulted in the best subjective image quality (Fig. 5).

\subsection{Objectively, PCA provides superior WM/GM separability}

The objective results agreed with the subjective observations discussed previously, the motion correction techniques are ranked by both the t-statistic and the difference of mean in the order: no correction, sensitivity, axial-COM, 3D-COM, PCA (Fig. 6). Interestingly, the two distributions from the PCA technique are actually more separable under a t-test than those from the no motion acquisition, although the distribution means are indeed further spread in the no motion acquisition. Motion correction was observed to have an effect similar to a local spatial filter, smoothing the image and thereby reducing the variance of the distribution (Fig. 5). This result does not imply that that motion corrected reconstructions are better than motion-free equivalent reconstructions. It may imply that the post-filtering applied in the no-motion case was lower than optimal, however, reconstruction parameters were held constant for consistency.

PCA, therefore, was determined to be most effective in this experiment at tracking rigid motion of a phantom. This technique was originally designed for periodic motion, such as respiratory motion [13]. Although it has been verified to be applicable to rigid motion previously [2], the technique has not been directly compared with alternatives for tracking rigid motion.

\subsection{Limitations and future opportunities}

The 3D-COM extension of the axial-COM technique is similar in concept to the COD technique 10 , 11]. When ToF measurement is available during the PET acquisition, the COD technique is likely to supply superior SNR. This is because the ToF information that is considered by COD but not 3D-COM provides superior localisation, and also because the 3D-COM technique only considers a subset of available data. However, when ToF measurement is not available, as is the case on the 
Siemens Biograph mMR, then the 3D-COM technique provides a useful approximation.

This investigation compared techniques for tracking motion for a simple translation of a phantom. This represents a simple approximation to a real neurological scan. Several variables that would be present in a human subject scan are not present, such as tracer dynamics. Additionally, in most neurological scans, a portion of the neck is in the field of view (FOV), meaning that some of the activity distribution moves with the head, and some does not. Such factors are likely to disrupt the motion surrogate extraction techniques to varying amounts. A future investigation should validate the comparison using clinical data.

No literature exists on optimising the parameters for the PCA technique. Parameters used in this investigation, such as the PCA preprocessing, the number of clusters extracted, and the number of motion gates during k-means clustering, were chosen to provide a good base-level performance, but are not optimal. Future work could investigate the optimisation of these parameters, and suggest good default choices for users.

\section{Conclusion}

PCA was demonstrated to be most effective for tracking rigid motion is this phantom experiment. The sensitivity and axial-COM techniques are mostly sensitive to axial motion, and so were ineffective in this phantom experiment. 3D-COM demonstrates improved transaxial motion sensitivity compared to axial-COM, but not to the level of effectiveness of PCA.

\section{Acknowledgements}

The authors thank the Herston Imaging Research Facility (HIRF) for providing scanner time, and the HIRF staff including Paul Thomas and Louise Campbell.

\section{Declarations}

Funding AGG was supported by an Australian Government Department of Education and Training Research Training Program stipend and a Commonwealth Scientific and Industrial Research Organisation (CSIRO) Top Up Scholarship when undertaking this work. 
Conflicts of interest There are no conflicts of interest to report.

Availability of data and material The data and materials are not available.

Code availability The code is not available.

Authors' contributions AGG, JS and ND conceived and ran the experiment and contributed to analysis and writing of the manuscript. SR advised on the experiment and contributed to analysis and writing of the manuscript. JAD contributed to analysis and writing of the manuscript.

Ethics approval Not applicable, no human data was used in this study.

Consent to participate Not applicable, no human data was used in this study.

Consent for publication Not applicable, no human data was used in this study.

\section{References}

[1] Huang C, Petibon Y, Normandin M, Li Q, Fakhri GE, Ouyang J. Fast head motion detection using PET list-mode data. Journal of Nuclear Medicine. 2015; 56:1827-1827.

[2] Thielemans K, Schleyer P, Dunn J, Marsden PK, Manjeshwar RM. Using PCA to detect head motion from PET list mode data. Nuclear Science Symposium and Medical Imaging Conference. Oct. 2013:1-5. DOI: 10.1109/NSSMIC. 2013.6829254.

[3] Ren S, Lu Y, Bertolli O, Thielemans K, Carson RE. Event-by-event non-rigid data-driven PET respiratory motion correction methods: comparison of principal component analysis and centroid of distribution. Physics in Medicine \& Biology. 2019; 64.16. Publisher: IOP Publishing:165014. DOI: $10.1088 / 1361-6560 / \mathrm{abObc9}$

[4] Visvikis D, Barret O, Fryer T, Turzo A, Lamare F, Rest CCL, Bizais Y. A posteriori respiratory motion gating of dynamic PET images. Nuclear Science Symposium and Medical Imaging Conference. Vol. 5. Oct. 2003:3276-3280 Vol.5. DOI: 10.1109/NSSMIC.2003.1352596.

[5] He J, O’Keefe GJ, Jones G, Saunder T, Gong SJ, Geso M, Scott AM. Evaluation of Geometrical Sensitivity for Respiratory Motion Gating by GATE and NCAT Simulation. 29th Annual International Conference of the IEEE Engineering in Medicine and Biology Society. Aug. 2007:4165-4168. DOI: 10.1109/IEMBS.2007.4353254. 
[6] Schleyer PJ, O'Doherty MJ, Barrington SF, Marsden PK. Retrospective data-driven respiratory gating for PET/CT. Physics in Medicine and Biology. 2009; 54.7:1935-1950. DOI: 10.1088/0031-9155/54/7/005.

[7] Schleyer PJ, Thielemans K, Marsden PK. Extracting a respiratory signal from raw dynamic PET data that contain tracer kinetics. Physics in Medicine and Biology. 2014; 59.15:43454356. DOI: $10.1088 / 0031-9155 / 59 / 15 / 4345$.

[8] Büther F, Dawood M, Stegger L, Wübbeling F, Schäfers M, Schober O, Schäfers KP. List Mode-Driven Cardiac and Respiratory Gating in PET. Journal of Nuclear Medicine. 2009; 50.5:674-681. DOI: $10.2967 /$ jnumed.108.059204.

[9] Feng T, Wang J, Sun Y, Zhu W, Dong Y, Li H. Self-Gating: An Adaptive Center-of-Mass Approach for Respiratory Gating in PET. IEEE Transactions on Medical Imaging. 2018; 37.5:1140-1148. DOI: 10.1109/TMI . 2017.2783739.

[10] Ren S, Jin X, Chan C, Jian Y, Mulnix T, Liu C, Carson RE. Data-driven respiratory motion estimation and correction using TOF PET list-mode centroid of distribution. IEEE Nuclear Science Symposium and Medical Imaging Conference (NSS/MIC). Nov. 2014:1-5. DOI: 10. 1109/NSSMIC. 2014.7431029.

[11] Ren S, Jin X, Chan C, Jian Y, Mulnix T, Liu C, Carson RE. Data-driven event-by-event respiratory motion correction using TOF PET list-mode centroid of distribution. Physics in Medicine \& Biology. 2017; 62.12:4741. DOI: 10.1088/1361-6560/aa700c.

[12] Schleyer PJ, Dunn JT, Reeves S, Brownings S, Marsden PK, Thielemans K. Detecting and estimating head motion in brain PET acquisitions using raw time-of-flight PET data. Physics in Medicine and Biology. 2015; 60.16:6441-6458. DOI: 10.1088/0031-9155/60/16/6441.

[13] Thielemans K, Rathore S, Engbrant F, Razifar P. Device-less gating for PET/CT using PCA. Nuclear Science Symposium and Medical Imaging Conference. Oct. 2011:3904-3910. DOI: 10 . 1109/NSSMIC. 2011.6153742.

[14] Pearson K. On lines and planes of closest fit to systems of points in space. The London, Edinburgh, and Dublin Philosophical Magazine and Journal of Science. 1901; 2.11:559-572. DOI: $10.1080 / 14786440109462720$. 
[15] Picard Y, Thompson CJ. Motion correction of PET images using multiple acquisition frames. IEEE Transactions on Medical Imaging. 1997; 16.2:137-144. DOI: 10.1109/42.563659.

[16] Dawood M, Lang N, Schafers KP. Lung motion correction on respiratory gated 3-D PET/CT images. IEEE Transactions on Medical Imaging. 2006; 25.4:476-485. DOI: 10.1109/TMI. 2006. 870892

[17] Freeman MF, Tukey JW. Transformations Related to the Angular and the Square Root. The Annals of Mathematical Statistics. 1950; 21.4:607-611. DOI: 10.1214/aoms/1177729756.

[18] Rivest-Hénault D, Dowson N, Greer PB, Fripp J, Dowling JA. Robust inverse-consistent affine CT-MR registration in MRI-assisted and MRI-alone prostate radiation therapy. Medical Image Analysis. 2015; 23.1:56-69. DOI: 10.1016/j.media.2015.04.014. 


\section{Figures}
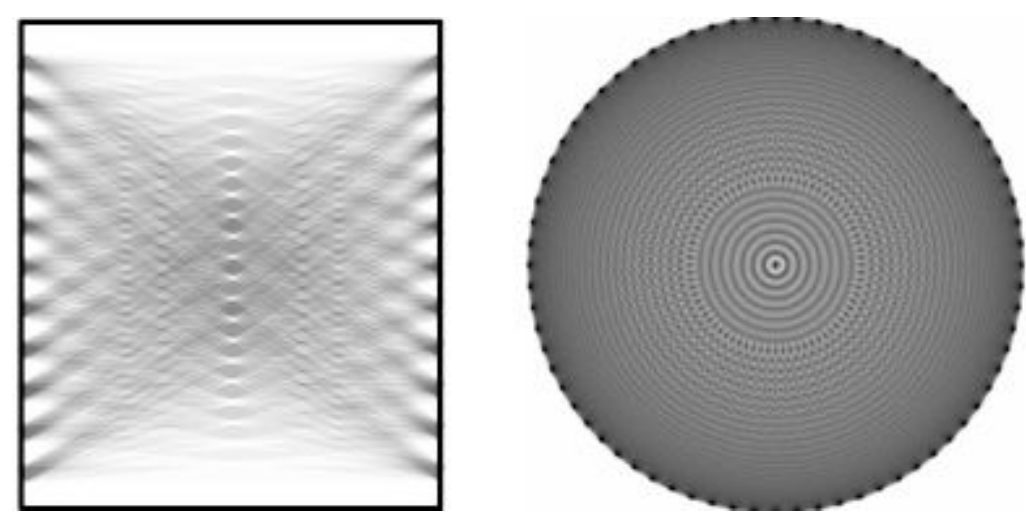

Figure 1

Left) Top/axial view, with the gantry axis running vertically, and Right) front/transaxial view of a cylindrical PET gantry. Lines of responses are shaded in with opacity so that darkness in the image approximates the spatial sensitivity.
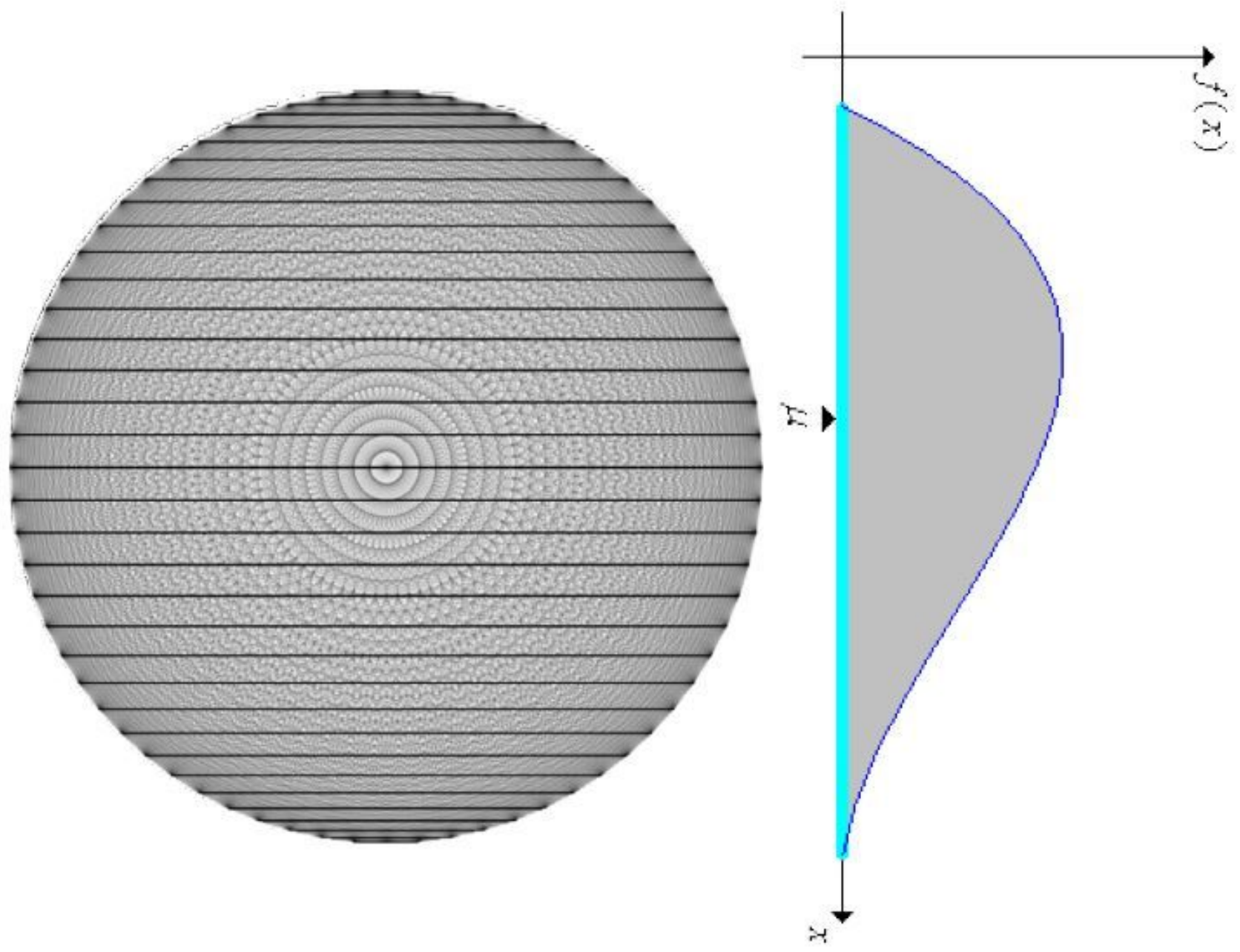

Figure 2 
An axial view of a gantry, with LORs shown in grey and horizontal LORs highlighted in black. The distribution of counts of the horizontal LORs gives an indication of the vertical distribution of the emission distribution.

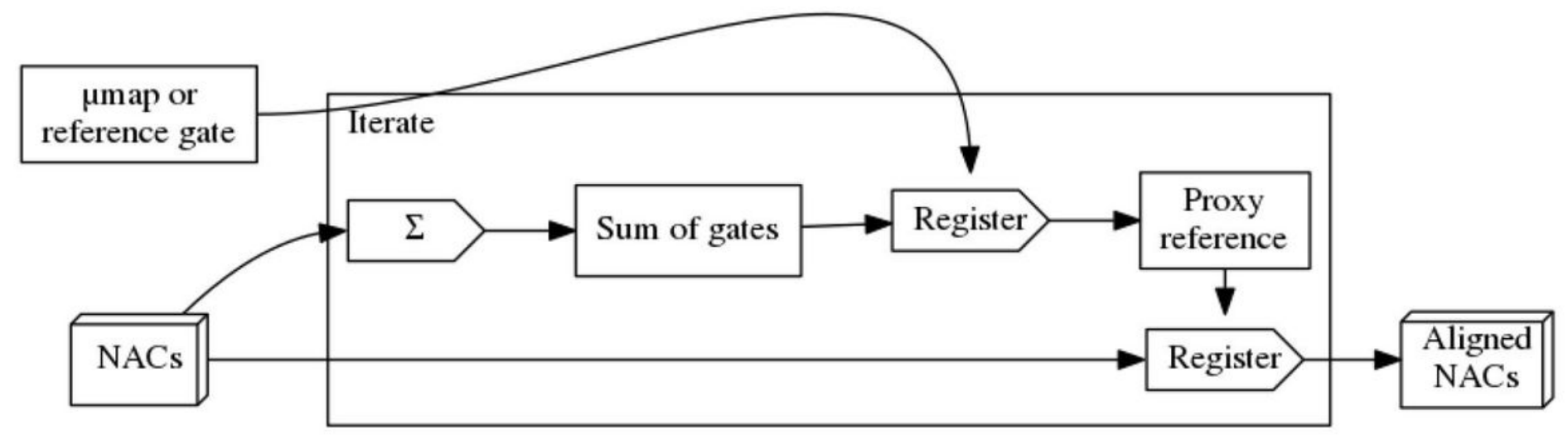

Figure 3

Iterative PET gate registration scheme. Square and cube shaped boxes indicate images and image series respectively. Pentagonal boxes indicate processes. In registration processes, the top input is the $\llbracket x e d /$ reference image, and the left input is the moving image. The portion of the work $\$ ow within the iterate box is repeated. 

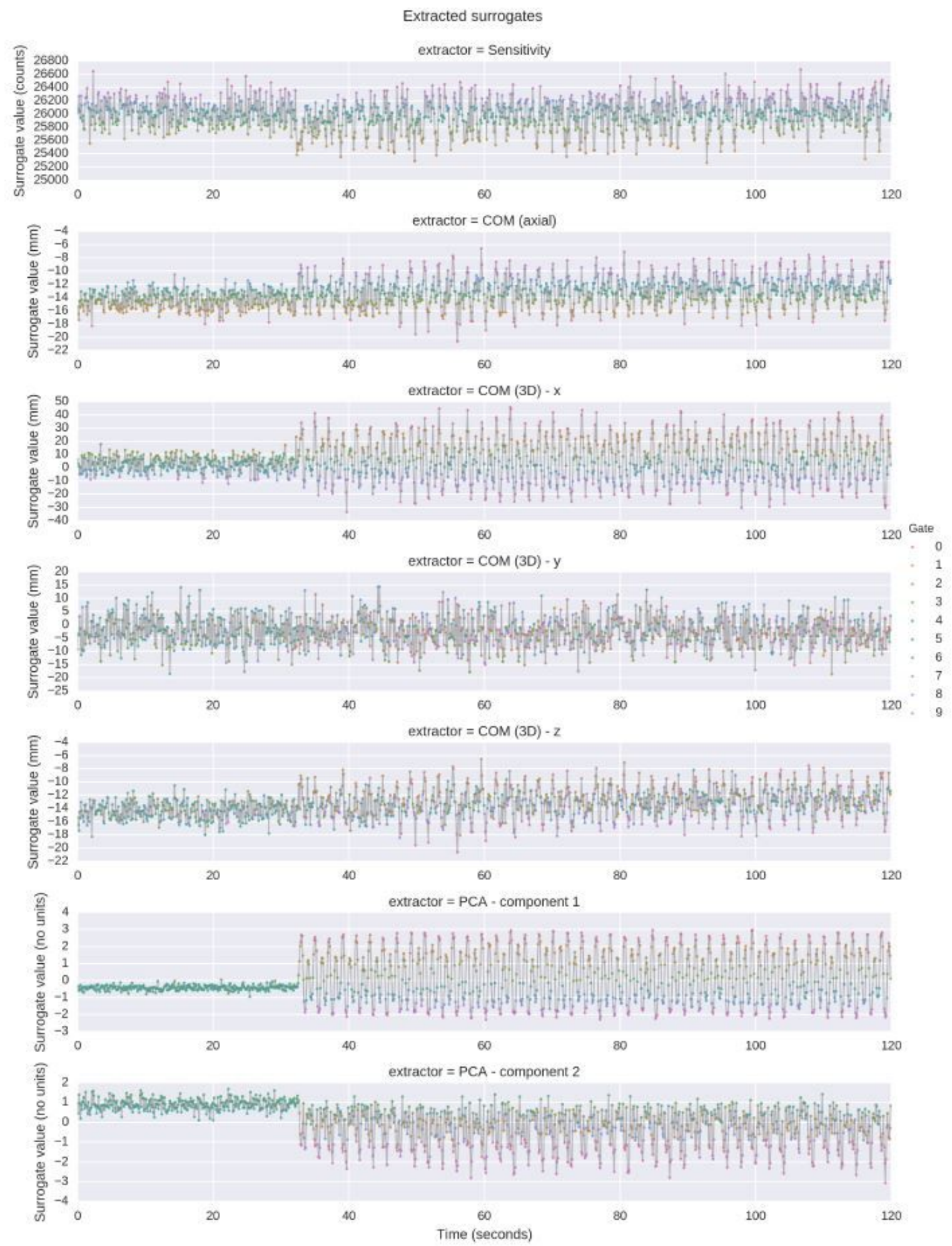

\section{Figure 4}

Plot of extracted surrogates. Surrogates have been gated, indicated by colour (online version only), using k-means gating. 

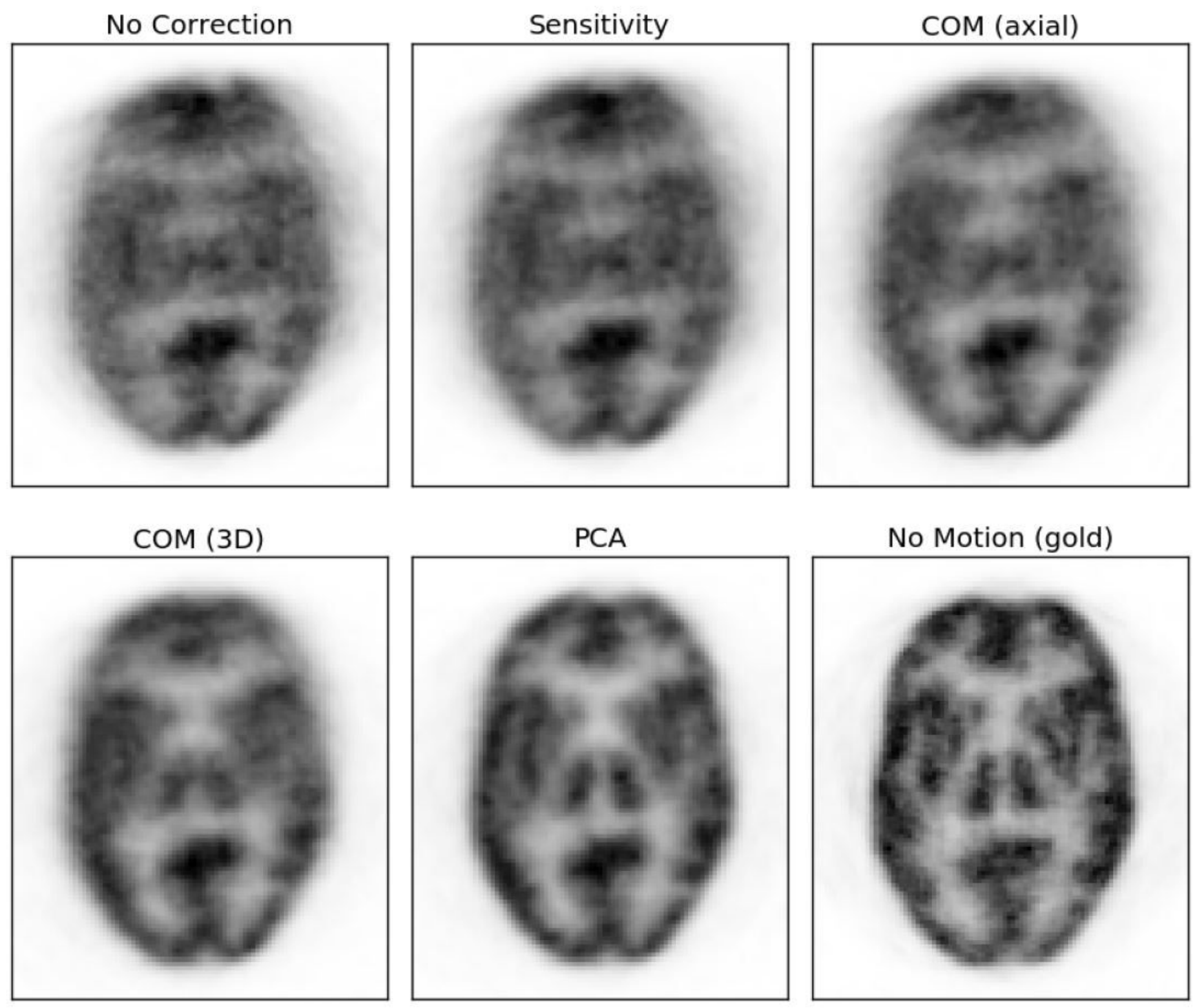

\section{Figure 5}

Axial slice of reconstructions with each of the investigated tracking techniques, as well as a reconstruction with no correction and a reconstruction of the reference acquisition of the phantom with no induced motion. 
Grey matter - white matter voxel intensity distributions

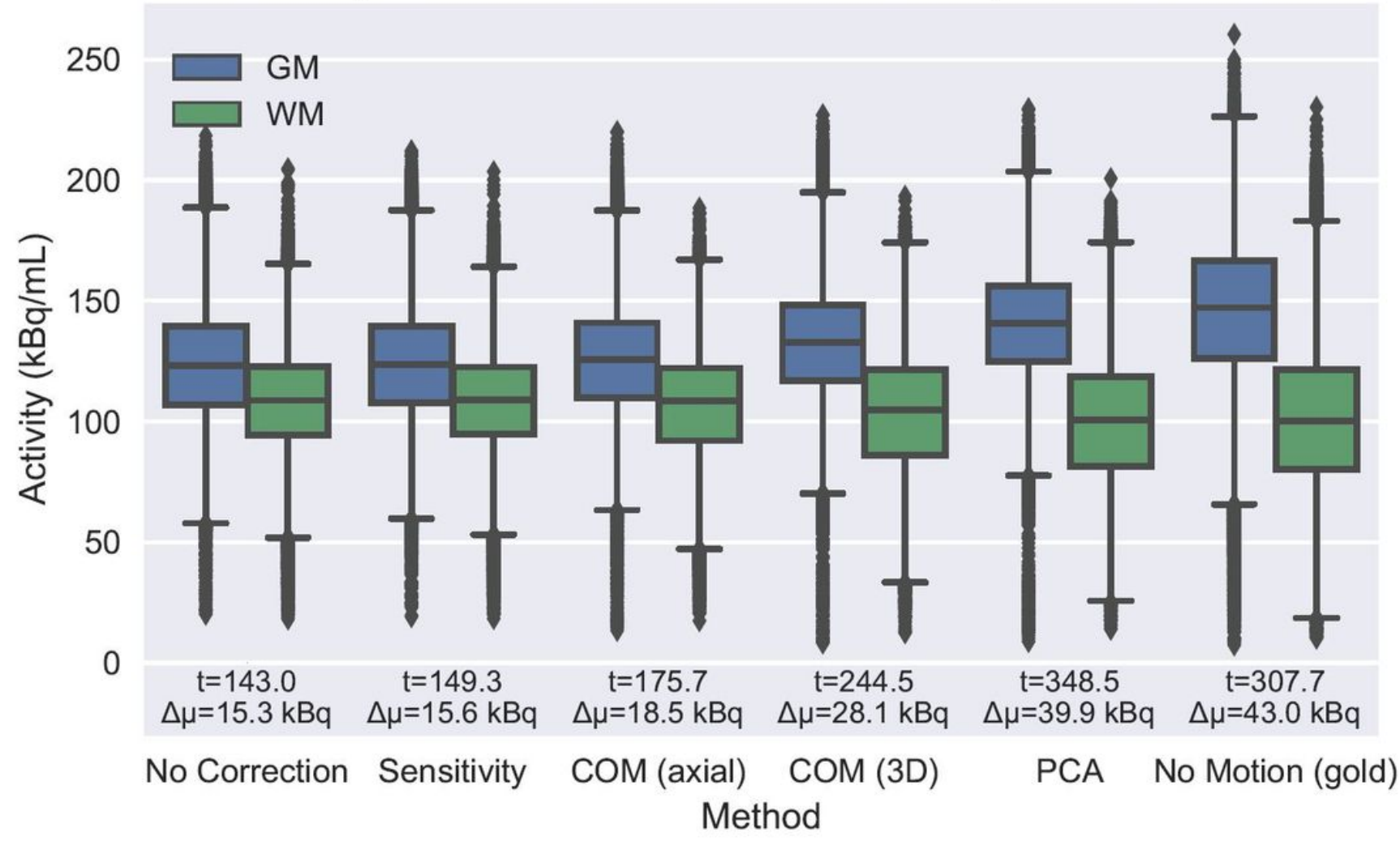

Figure 6

Distributions of activity in voxels labelled GM (left and blue online) and WM (right and green online) in the CT segmentation, for each correction method investigated. Shown below each pair of distributions is the $\mathrm{t}$-statistic between them $(\mathrm{t})$ and the difference in the distribution means $(\Delta \mu)$. A larger metric implies a greater separation between classes, indicating less blurring. 